\title{
A Review of the Ultrathin Orsiro Biodegradable Polymer Drug-eluting Stent in the Treatment of Coronary Artery Disease
}

\author{
James J Wu, ${ }^{1,2}$ Joshua AH Way, ${ }^{1}$ David Brieger ${ }^{1,2}$ \\ 1. Sydney Medical School, The University of Sydney, Camperdown, Australia; 2. Department of Cardiology, Concord Repatriation General \\ Hospital, Concord, Australia
}

$\mathrm{D}$ rug-eluting stents (DES) have revolutionised the treatment of coronary artery disease (CAD) in patients undergoing percutaneous coronary intervention. In recent years, there has been a focus on a new generation of DES, such as biodegradable polymer DES (BP-DES). This novel stent platform was developed with the hope of eliminating the risk of very late stent thrombosis associated with the current gold-standard durable polymer DES (DP-DES). Ultrathin Orsiro BP-DES (Biotronik, Bülach, switzerland) are based on a cobalt-chromium stent platform that is coated with a bioresorbable polymer coating containing sirolimus. These devices have one of the thinnest struts available in the current market and have the theoretical benefit of reducing a chronic inflammatory response in the vessel wall. In 2019, the United States Food and Drug Administration (FDA) approved the use of Orsiro BP-DES in patients with CAD based on promising results in recent landmark trials, such as BIOFLOWV and BIOSTEMI. The aim of the present review article was to discuss the history of stent technology and the continued opportunities for improvements, focusing on the potential benefits of Orsiro BP-DES.

\section{Keywords}

Biodegradable polymer, coronary artery disease, drug-eluting stents, Orsiro

Disclosure: James J Wu, Joshua AH Way and David Brieger have nothing to declare in relation to this article. Review Process: Double-blind peer review.

Compliance with Ethics: This article involves a review of literature and does not report on new clinical data, or any studies with human or animal subjects performed by any of the authors.

Authorship: The named authors meet the International Committee of Medical Journal Editors (ICMJE) criteria for authorship of this manuscript, take responsibility for the integrity of the work as a whole, and have given final approval for the version to be published.

Received: 9 September 2019

Accepted: 25 November 2019

Citation: Heart International. 2019;13(2):17-24

Corresponding Author: David Brieger, Professor in Cardiology, The University of Sydney, NSW 2006, Australia. E: david.brieger@health.nsw.gov.au

Support: No funding was received in the publication of this article.
Coronary artery disease (CAD) is a major global cause of death attributing to nearly 9 million deaths worldwide in 2016. ${ }^{1}$ The introduction of percutaneous coronary intervention (PCI) has revolutionised the treatment of CAD in current clinical practice, and advances in coronary stent technology have continued to deliver improved outcomes. In recent years, there has been a focus on a new generation of drug-eluting stents (DES), such as biodegradable polymer DES (BP-DES). This novel stent platform utilises a bioresorbable polymer coating that provides controlled release of an anti-proliferative drug.

A number of innovations in stent design have been made to address the risk of very late stent thrombosis (ST) associated with the current gold-standard durable polymer DES (DP-DES). In 2019, the US Food and Drug Administration (FDA) approved the use of ultrathin Orsiro BP-DES (Biotronik, Bülach, Switzerland) in patients with CAD. ${ }^{2} \mathrm{New}$-generation DES have demonstrated promising results in landmark trials. The aim of this review article was to discuss the history of stent technology and the continued opportunities for improvements, focusing on the potential benefits of Orsiro BP-DES.

\section{Percutaneous transluminal coronary angioplasty}

Coronary stent technology has made remarkable progress over the last few decades in terms of stent design, structure, and component materials. ${ }^{3}$ Percutaneous transluminal coronary angioplasty (PTCA) was first performed in 1977 by Grüntzig et al. ${ }^{4}$ As a minimally invasive procedure, PTCA offers an alternative revascularisation strategy to coronary artery bypass grafting, especially for patients deemed unsuitable for surgery. However, during PTCA, balloon dilation causes atherosclerotic plaque disruption and endothelial denudation, which provoke vascular injury and inflammation. ${ }^{5}$ This mechanical trauma also induces various processes, such as elastic recoil, intimal dissection, and neointimal hyperplasia. ${ }^{6}$ These factors contribute to abrupt vessel closure and restenosis, which are limitations of PTCA. ${ }^{7}$ Abrupt vessel closure refers to sudden coronary occlusion after $\mathrm{PCl}$ leading to myocardial infarction (MI). The incidence of abrupt vessel closure within 24 hours of PTCA was reported to be $8 \% .{ }^{8}$ Restenosis is defined as $>50 \%$ reduction in lumen diameter after PCI. ${ }^{9}$ The incidence of restenosis within 6 months of PTCA was reported to be $40 \% .{ }^{10}$

\section{Bare metal stents}

Bare metal stents (BMS) were developed to address the limitations of PTCA. These devices are a stainless steel mesh tube that is delivered and expanded by a balloon-tipped catheter to optimise the lumen of the diseased vessel. ${ }^{11}$ In current clinical practice, the majority of PCl procedures involve both angioplasty and stent placement. BMS implantation was first performed in 1986 by Sigwart et al. ${ }^{12}$ The stent acts as a scaffold that prevents abrupt vessel closure and 
restenosis by tacking intimal dissection flaps against the vessel wall and maintaining the widened lumen diameter. ${ }^{13}$ Intravascular ultrasound was first introduced in the 1980s, and is routinely used in high-pressure balloon dilation to enhance stent expansion and strut apposition to the vessel wall. ${ }^{14}$ Optical coherence tomography is an emerging imaging modality that can be applied as a periprocedural tool for stent planning, improving lumen assessment and plaque characterisation compared to intravascular ultrasound. ${ }^{15}$ In current clinical practice, the use of intravascular imaging has been a major determinant in optimising stent expansion and placement.

In 1994, the FDA approved the use of BMS in patients with CAD. ${ }^{16}$ The BENESTENT trial found that BMS had a significantly reduced rate of restenosis at 7 months compared with PTCA (22\% versus 32\%; $\mathrm{p}=0.02) .{ }^{17}$ However, BMS were hindered by the risk of in-stent restenosis (ISR). During BMS implantation, stent expansion and strut apposition to the vessel wall provoke vascular injury and inflammation. ${ }^{18}$ This mechanical trauma leads to neointimal hyperplasia and gradual narrowing of the lumen. ${ }^{19}$ The incidence of ISR was reported to range from $17 \%$ to $41 \% .^{20}$

\section{First-generation durable polymer drug-eluting stents}

DP-DES were introduced to overcome the risk of ISR associated with BMS. These devices are based on a stainless steel stent platform that is coated with a permanent polymer containing an anti-proliferative drug. ${ }^{21}$ The permanent polymer coating provides controlled release of the anti-proliferative drug over 4-6 weeks following DP-DES implantation, to inhibit neointimal hyperplasia and theoretically eliminate the risk of ISR associated with BMS.22 DP-DES implantation was first performed in 1999 by Sousa et al. ${ }^{23}$ First-generation DP-DES release sirolimus or paclitaxel, both of which are effective agents for modifying intracellular signalling to prevent neointimal hyperplasia. ${ }^{24}$ Sirolimus is an immunosuppressive macrolide extracted from the bacterium Streptococcus hygroscopicus that prevents vascular smooth muscle cell proliferation by inhibiting mammalian target of rapamycin (mTOR) and arresting the cell cycle progression from $\mathrm{G} 1$ to $\mathrm{S}$ phase. ${ }^{25}$ Paclitaxel is an antineoplastic drug extracted from the yew Taxus brevifolia. It prevents vascular smooth muscle cell proliferation by stabilising microtubules and arresting the cell cycle progression from G2 to M phase. ${ }^{26}$ The RAVEL trial found that sirolimus-eluting stents (SES) had a significantly reduced rate of ISR at 6 months compared with BMS (0\% versus $26.6 \%$; $p<0.001) .{ }^{27}$ Similarly, the TAXUS I trial found that paclitaxel-eluting stents (PES) had significantly reduced in-stent late lumen loss at 12 months compared with BMS $(0.36 \pm 0.48$ versus $0.71 \pm 0.48 \mathrm{~mm} ; \mathrm{p}<0.01) .{ }^{28}$ In-stent late lumen loss is defined as the difference in minimum lumen diameter within the stented segment immediately after $\mathrm{PCl}$ and at a specified follow-up period. ${ }^{29}$ This angiographic measurement is used as a surrogate marker to estimate the risk of ISR ${ }^{30}$ The FDA approved the use of SES and PES in 2003 and 2004, respectively.31

\section{Stent thrombosis}

Despite resolving the limitations of BMS, first-generation DP-DES were impeded by the risk of ST. This adverse event can be classified according to the probability of event. According to the Academic Research Consortium, ${ }^{32}$ definite ST is defined by either angiographic or pathological evidence of ST, probable ST is defined as either any unexplained death within 30 days of stent placement or any $\mathrm{Ml}$ within the territory of implanted stent, and possible ST is defined as any unexplained death beyond 30 days. ST can also be classified according to the timing of event following stent placement. Acute ST occurs within 24 hours, subacute ST occurs between 1 and 30 days, late ST occurs beyond 30 days, and very late ST occurs beyond 12 months. ${ }^{33}$

The mechanism of ST is attributed to the permanent polymer coating that triggers a chronic inflammatory response in the vessel wall involving incomplete re-endothelialisation, neoathersclerosis, and hypersensitivity reaction. ${ }^{34,35}$ Sirolimus and paclitaxel in first-generation DP-DES are also toxic agents that potentiate the expression of tissue factor in endothelial cells and form a pro-thrombogenic environment leading to ST. ${ }^{36}$ A challenge faced by novel stent platforms is the development of less toxic anti-proliferative drugs that provide an adequate balance between the inhibition of neointimal hyperplasia and the stimulation of early re-endothelialisation. There have been concerns about the safety profile of DP-DES, with the risks of late and very late ST. A pooled analysis of 1,748 patients found that there was a significantly increased rate of very late ST at 4 years for SES ( $0.6 \%$ versus $0 \% ; p=0.025)$ and PES $(0.7 \%$ versus $0.2 \% ; \mathrm{p}=0.028)$ compared with $\mathrm{BMS}^{3{ }^{37}}$ The median timing of very late ST for SES and PES was reported to be 15.5 months and 18.0 months, respectively. ${ }^{38}$

\section{Second-generation durable polymer drug-eluting stents}

Second-generation DP-DES were developed to address the risk of very late ST associated with first-generation SES and PES. These devices are based on a similar structure to the preceding generation, but utilise more biocompatible polymer coatings, less toxic anti-proliferative drugs, and thinner metal alloy stent (cobalt-chromium or platinum-chromium) platforms. ${ }^{39}$ Second-generation DP-DES release everolimus or zotarolimus, both of which are sirolimus analogues with shorter half-lives and fewer collateral effects. ${ }^{40}$ The COMPARE trial found that everolimus-eluting stents (EES) had a significantly reduced rate of definite or probable ST at 5 years compared with PES $(3.1 \%$ versus 5.9\%; $p=0.005) .{ }^{41}$ Similarly, the ENDEAVOR IV trial found that zotarolimus-eluting stents (ZES) had a significantly reduced rate of very late ST at 5 years compared with PES (0.4\% versus $1.8 \% ; p=0.012){ }^{42}$ The FDA approved the use of EES and ZES in patients with CAD in 2008 and 2012, respectively. ${ }^{43}$ Second-generation DP-DES have largely replaced the preceding generation of devices, and are recognised as the current gold standard for $\mathrm{PCl}{ }^{44}$ However, the issue of very late ST persisted in second-generation DP-DES. The incidence of very late ST over 5 years for EES and ZES was reported to be $1.5 \%$ and $1.3 \%$, respectively. ${ }^{45}$ Despite the use of more biocompatible materials, the permanent polymer coating in second-generation DP-DES remains a driving factor for very late ST.

\section{Biodegradable polymer drug-eluting stents}

The ongoing issue of very late ST associated with DP-DES has led to the advent of BP-DES. These new-generation DES are based on a metallic stent platform that is coated with a biodegradable polymer releasing an anti-proliferative drug. ${ }^{46}$ The degradation of the polymer coating removes the potential stimulus for a chronic inflammatory response in the vessel wall, and theoretically eliminates the risk of very late ST associated with DP-DES. The polymer coating of BP-DES dissolves over time following stent placement, during which there is controlled release of the anti-proliferative drug. ${ }^{47}$ The metallic stent platform remains in the vessel once the polymer coating has completely degraded. BP-DES release everolimus, sirolimus, or biolimus. Biolimus is a highly lipophilic sirolimus analogue that easily crosses the cell membrane to achieve rapid onset of action and minimal collateral effects. ${ }^{48}$ In 2019, the FDA approved the use of ultrathin Orsiro BP-DES in patients with CAD, based on promising results in recent landmark trials. ${ }^{2}$ Table 1 summarises the characteristics of current DES. 
Table 1: Characteristics of current drug-eluting stents

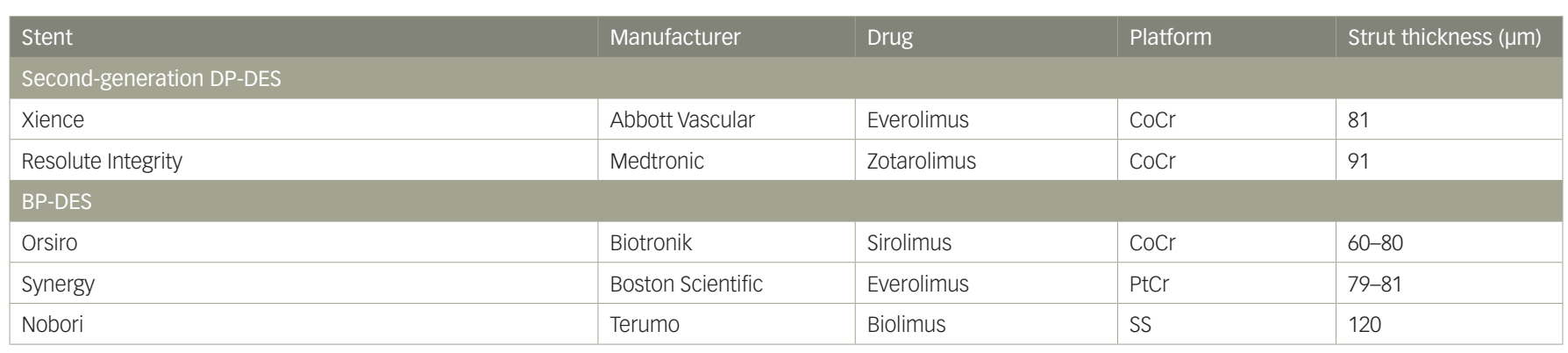

BP-DES = biodegradable polymer drug-eluting stents; $C O C r=$ cobalt-chromium; DP-DES = durable polymer drug-eluting stents; PtCr = platinum-chromium; SS = stainless steel.

\section{Strut thickness}

The importance of strut thickness to the clinical performance of DES has been demonstrated in several studies. An ex vivo study involving flow loops showed that thick-strut $(162 \mu \mathrm{m})$ stents had 1.5-fold increase in thrombogenicity at 3 days compared with thin-strut $(81 \mu \mathrm{m})$ stents $(p<0.001) .{ }^{49}$ An animal study involving rabbit denudation models showed that thin-strut $(81 \mu \mathrm{m})$ stents had significantly increased strut tissue coverage at 14 days compared with thick-strut $(132 \mu \mathrm{m})$ stents (77 $\pm 6 \%$ versus $95 \pm 4 \% ; p=0.001) .{ }^{50}$ The thinner struts promote early re-endothelialisation and arterial healing due to the smaller area requiring neointimal tissue coverage. ${ }^{51}$ The ISAR-STEREO trial found that thin-strut $(50 \mu \mathrm{m})$ stents had significantly reduced rates of ISR at 6 months compared with thick-strut $(140 \mu \mathrm{m})$ stents $(15.0 \%$ versus 25.8\%; $p=0.003$ ). ${ }^{52}$ The thinner struts have less traumatic effect resulting in reduced vascular injury, inflammation and disruption of local blood flow. ${ }^{51}$ The thinner struts also enable greater flexibility for stent delivery through calcified or tortuous vessels. ${ }^{53}$

Orsiro BP-DES have one of the thinnest struts available in the current market, with a strut thickness of either $60 \mu \mathrm{m}$ for the $2.25-3.00 \mathrm{~mm}$ stent platform or $80 \mu \mathrm{m}$ for the $3.50-4.00 \mathrm{~mm}$ stent platform. ${ }^{54}$ Nobori ${ }^{\circledast}$ BP-DES (Terumo, Tokyo, Japan) are similar devices, with a strut thickness of $120 \mu \mathrm{m} .{ }^{55}$ The SORT OUT VII trial found that Orsiro BP-DES had a significantly reduced risk of definite ST at 12 months compared with Nobori BP-DES (0.4\% versus 1.2\%; $\mathrm{p}=0.03) .{ }^{56}$ The investigators suggested that thinner struts have the potential to reduce thrombogenicity and thrombus mobilisation.

\section{Dual antiplatelet therapy}

The introduction of dual antiplatelet therapy (DAPT) led to a reduced risk of ST associated with DES. DAPT has been adopted as the routine antithrombotic regimen following stent placement, and consists of aspirin plus adenosine diphosphate $\left(\mathrm{P} 2 \mathrm{Y}_{12}\right)$ receptor inhibitor. ${ }^{57}$ According to the latest American College of Cardiology/American Heart Association guidelines, the recommended duration of DAPT following DP-DES implantation is 12 months, with lifelong continuation of aspirin. ${ }^{58}$ Short-term DAPT (6 months) has been proposed for BP-DES implantation due to improved arterial healing in the stented segment. The NIPPON trial found that 6-month and 18-month DAPT conferred equivocal safety and efficacy profiles in patients receiving BP-DES, with comparable rates of ST $(0.1 \%$ versus $0.1 \% ; p=1.00)$, cardiac death $(0.2 \%$ versus $0.5 \% ; p=0.39)$, and all-cause death $(0.4 \%$ versus $1.0 \% ; p=0.09) .{ }^{59}$ Patients with poor compliance or high bleeding risk might theoretically benefit from BP-DES due to the shortened duration of DAPT.
Figure 1: Features of Orsiro biodegradable polymer drug-eluting stents

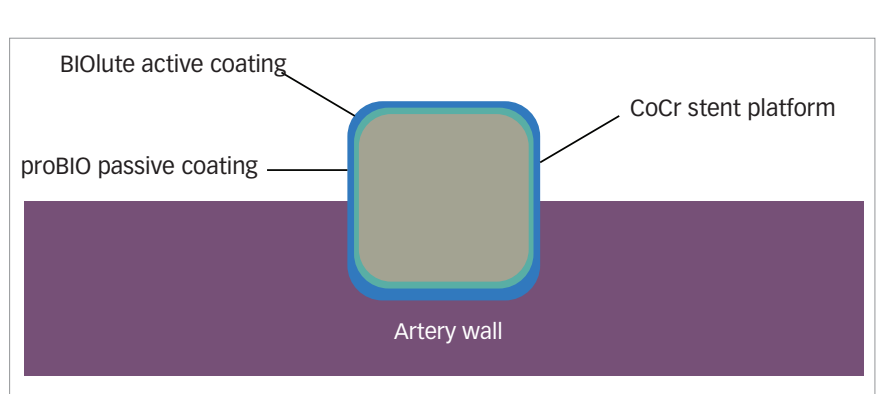

BIOlute = bioabsorbable poly-L-lactide eluting a limus drug; $\mathrm{CoCr}=$ cobalt chromium; proBIO = amorphous silicon carbide

\section{Ultrathin Orsiro biodegradable polymer drug-eluting stents}

Ultrathin Orsiro BP-DES are based on a cobalt-chromium stent platform in a double-helix structure, with helical meanders, longitudinal connectors, and wedge-shaped transitions..$^{60}$ The metal alloy cobalt-chromium allows for thinner struts, which provide greater flexibility for effective stent delivery. ${ }^{61}$ During stent expansion, the double-helix structure adapts to the vessel curvature without unnatural straightening. ${ }^{62}$ This design feature also provides high radial strength to prevent longitudinal compression and foreshortening throughout the entire length of the stent. ${ }^{63}$

Orsiro BP-DES have a hybrid coating consisting of active and passive components to improve biocompatibility (Figure 1). The outer layer contains a BIOlute active coating (Biotronik, Bülach, Switzerland) that is made from biodegradable poly-L-lactic acid (PLLA) loaded with sirolimus. ${ }^{64}$ The PLLA undergoes metabolism via the Krebs cycle into carbon dioxide and water, and completely degrades over 12-15 months. ${ }^{65}$ The slow degradation of PLLA allows for the controlled release of sirolimus, since the drug elution period is shorter than the polymer degradation period. The release of sirolimus occurs at a dose of $1.4 \mu \mathrm{g} / \mathrm{mm}^{2}$ over 3 months. ${ }^{66}$ A histopathology study showed that PLLA SES had a significantly reduced neointimal area at 180 days compared with durable polymer SES $\left(1.8 \pm 1.2\right.$ versus $\left.3.0 \pm 1.5 \mathrm{~mm}^{2} ; \mathrm{p}=0.01\right)$. The investigators also reported that PLLA SES had sufficient sirolimus vascular tissue concentration, reduced inflammation, and improved re-endothelialisation compared with durable polymer SES. Furthermore, the BIOlute active coating has an abluminal thickness of $7.5 \mu \mathrm{m}$ and a luminal thickness of $3.5 \mu \mathrm{m} .{ }^{67}$ This asymmetric circumferential distribution ensures adherence of the polymer coating to the stent platform in 
Table 2: Findings of clinical studies related to Orsiro biodegradable polymer drug-eluting stents

\begin{tabular}{|c|c|c|c|c|c|c|c|c|}
\hline \multirow[t]{2}{*}{ Study name } & \multirow[t]{2}{*}{ Year } & \multirow[t]{2}{*}{ Study type } & \multirow{2}{*}{$\begin{array}{l}\text { Follow-up } \\
\text { (months) }\end{array}$} & \multirow{2}{*}{$\begin{array}{l}\text { Stent } \\
\text { comparator } \\
\text { type }\end{array}$} & \multicolumn{2}{|c|}{ Patients (n) } & \multirow[t]{2}{*}{ Outcomes } & \multirow[t]{2}{*}{ Conclusion } \\
\hline & & & & & $\begin{array}{l}\text { Orsiro } \\
\text { BP-DES }\end{array}$ & $\begin{array}{l}\text { Stent } \\
\text { comparator }\end{array}$ & & \\
\hline BIOFLOW $\|^{76}$ & 2015 & $\begin{array}{l}\text { Non-inferiority } \\
\text { RCT }\end{array}$ & 12 & Xience EES & 298 & 154 & $\begin{array}{l}\text { - } \quad \text { Definite or probable ST: } 0 \% \text { versus } 0 \% \\
\text { - } \quad \text { MI: } 3.1 \% \text { versus } 2.6 \% ; p=0.80 \\
\text { - } \quad \text { Cardiac death: } 0.7 \% \text { versus } 0.7 \% ; p=0.98 \\
\text { - } \quad \text { All-cause death: } 1.0 \% \text { versus } 0.7 \% ; p=0.71 \\
\text { - } \quad \text { TLR: } 3.8 \% \text { versus } 5.4 \% ; p=0.46 \\
\text { - } \quad \text { In-stent LLL at } 9 \text { months: } 0.10 \pm 0.32 \mathrm{~mm} \\
\quad \text { versus } 0.11 \pm 0.29 \text { mm; } p=0.98\end{array}$ & Non-inferior \\
\hline $\begin{array}{l}\text { SORT OUT } \\
\text { VIII5 }\end{array}$ & 2016 & $\begin{array}{l}\text { Non-inferiority } \\
\text { RCT }\end{array}$ & 12 & $\begin{array}{l}\text { Nobori } \\
\text { BP-DES }\end{array}$ & 1,261 & 1,264 & $\begin{array}{l}\text { - } \quad \text { Definite ST: } 0.4 \% \text { versus } 1.2 \% ; p=0.03 \\
\text { - } \quad \text { Ml: } 1.6 \% \text { versus } 2.4 \% ; p=0.16 \\
\text { - } \quad \text { Cardiac death: } 1.3 \% \text { versus } 1.4 \% p=0.75 \\
\text { - } \quad \text { All-cause death: } 3.0 \% \text { versus } 2.2 \% ; p=0.21 \\
\text { - } \quad \text { TLR: } 2.0 \% \text { versus } 2.9 \% ; p=0.13\end{array}$ & $\begin{array}{l}\text { Non-inferior } \\
\text { Definite ST lower } \\
\text { with Orsiro } \\
\text { BP-DES than } \\
\text { Nobori BP-DES }\end{array}$ \\
\hline ORIENT 78 & 2017 & $\begin{array}{l}\text { Non-inferiority } \\
\text { RCT }\end{array}$ & 12 & $\begin{array}{l}\text { Resolute } \\
\text { Integrity ZES }\end{array}$ & 250 & 122 & $\begin{array}{l}\text { - } \quad \text { Definite or probable } \mathrm{ST} \text { : } 0 \% \text { versus } 0 \% \\
\text { - } \\
\text { - }\end{array}$ & Non-inferior \\
\hline PRISON IV ${ }^{79}$ & 2017 & $\begin{array}{l}\text { Non-inferiority } \\
\mathrm{RCT} \\
\text { (chronic total } \\
\text { occlusion) }\end{array}$ & 12 & Xience EES & 165 & 165 & $\begin{array}{l}\text { - } \quad \text { Definite or probable ST: } 0.6 \% \text { versus } 0.6 \% \\
\text { - } \quad \text { MI: } 0.6 \% \text { versus } 0.6 \% \\
\text { - } \quad \text { Cardiac death: } 0.6 \% \text { versus } 1.2 \% \\
\text { - } \quad \text { All-cause death: } 0.6 \% \text { versus } 1.8 \% \\
\text { - } \quad \text { TLR: } 10.5 \% \text { versus } 4.0 \% ; \mathrm{p}=0.04 \\
\text { - } \quad \text { In-stent LLL at } 9 \text { months: } 0.12 \pm 0.59 \mathrm{~mm} \\
\quad \text { versus } 0.07 \pm 0.46 \mathrm{~mm} ; \mathrm{p}=0.52\end{array}$ & $\begin{array}{l}\text { Non-inferior } \\
\text { TLR higher with } \\
\text { Orsiro BP-DES } \\
\text { than Xience EES }\end{array}$ \\
\hline BIOSCIENCE ${ }^{80}$ & 2018 & $\begin{array}{l}\text { Non-inferiority } \\
\text { RCT }\end{array}$ & 60 & Xience EES & 1,063 & 1,056 & $\begin{array}{l}\text { - } \quad \text { Definite or probable ST: } 6.3 \% \text { versus } 7.7 \% ; \\
\quad p=0.26 \\
\text { - } \quad \text { Ml: } 10.4 \% \text { versus } 12.3 \% ; p=0.23 \\
\text { - } \quad \text { Cardiac death: } 8.6 \% \text { versus } 7.5 \% ; p=0.57 \\
\text { - } \quad \text { All-cause death } 14.1 \% \text { versus } 10.3 \% ; p=0.02 \\
\text { - } \quad \text { TLR: } 11.5 \% \text { versus } 10.9 \% ; p=0.61\end{array}$ & $\begin{array}{l}\text { Non-inferior } \\
\text { All-cause death } \\
\text { higher with } \\
\text { Orsiro BP-DES } \\
\text { than Xience EES }\end{array}$ \\
\hline BIO-RESORT ${ }^{81}$ & 2018 & $\begin{array}{l}\text { Non-inferiority } \\
\text { RCT }\end{array}$ & 24 & $\begin{array}{l}\text { Resolute } \\
\text { Integrity ZES }\end{array}$ & 1,169 & 1,173 & $\begin{array}{l}\text { - } \quad \text { Definite or probable ST: } 0.6 \% \text { versus } 0.8 \% ; \\
\quad p=0.62 \\
\text { - } \quad \text { Ml: } 3.1 \% \text { versus } 3.6 \% ; p=0.50 \\
\text { - } \quad \text { Cardiac death: } 1.3 \% \text { versus } 1.5 \% ; p=0.73 \\
\text { - } \quad \text { All-cause death: } 2.6 \% \text { versus } 3.3 \% ; p=0.33 \\
\text { - } \quad \text { TLR: } 2.2 \% \text { versus } 3.0 \% ; p=0.24 \\
\text { - } \quad \text { TLR beyond } 12 \text { months: } 0.6 \% \text { versus } 1.5 \% ; \\
\quad p=0.04\end{array}$ & $\begin{array}{l}\text { Non-inferior } \\
\text { TLR beyond } 12 \\
\text { months lower } \\
\text { with Orsiro } \\
\text { BP-DES than } \\
\text { Resolute Integity } \\
\text { ZES }\end{array}$ \\
\hline BIONYX ${ }^{82}$ & 2018 & $\begin{array}{l}\text { Non-inferiority } \\
\text { RCT }\end{array}$ & 12 & $\begin{array}{l}\text { Resolute } \\
\text { Onyx ZES }\end{array}$ & 1,245 & 1,243 & $\begin{array}{l}\text { - } \quad \text { Definite or probable ST: } 0.7 \% \text { versus } 0.1 \% ; \\
\quad p=0.01 \\
\text { - } \quad \text { Ml: } 1.6 \% \text { versus } 1.6 \% ; p=0.97 \\
\text { - } \quad \text { Cardiac death: } 1.1 \% \text { versus } 0.6 \% ; p=0.18 \\
\text { - } \quad \text { All-cause death: } 2.1 \% \text { versus } 1.6 \% ; p=0.37 \\
\text { - } \quad \text { TLR: } 2.0 \% \text { versus } 2.5 \% ; p=0.35\end{array}$ & $\begin{array}{l}\text { Non-inferior } \\
\text { Definite or } \\
\text { probable ST } \\
\text { higher with } \\
\text { Orsiro BP-DES } \\
\text { than Resolute } \\
\text { Onyx ZES }\end{array}$ \\
\hline
\end{tabular}


Table 2: Cont.

\begin{tabular}{|c|c|c|c|c|c|c|c|c|}
\hline \multirow[t]{2}{*}{ Study name } & \multirow[t]{2}{*}{ Year } & \multirow[t]{2}{*}{ Study type } & \multirow{2}{*}{$\begin{array}{l}\text { Follow-up } \\
\text { (months) }\end{array}$} & \multirow{2}{*}{$\begin{array}{l}\text { Stent } \\
\text { comparator } \\
\text { type }\end{array}$} & \multicolumn{2}{|c|}{ Patients (n) } & \multirow[t]{2}{*}{ Outcomes } & \multirow[t]{2}{*}{ Conclusion } \\
\hline & & & & & $\begin{array}{l}\text { Orsiro } \\
\text { BP-DES }\end{array}$ & $\begin{array}{l}\text { Stent } \\
\text { comparator }\end{array}$ & & \\
\hline BIOFLOW V ${ }^{83}$ & 2018 & $\begin{array}{l}\text { Non-inferiority } \\
\text { RCT }\end{array}$ & 24 & Xience EES & 884 & 450 & $\begin{array}{l}\text { - } \quad \text { Definite very late } \mathrm{ST}: 0.1 \% \text { versus } 1.0 \% ; p=0.045 \\
\text { - } \quad \text { MI: } 5.3 \% \text { versus } 9.5 \% ; p=0.01 \\
\text { - } \quad \text { Cardiac death: } 0.6 \% \text { versus } 0.5 \% ; p=1.00 \\
\text { - } \quad \text { All-cause death: } 1.9 \% \text { versus } 2.2 \% ; p=0.83 \\
\text { - } \quad \text { TLR: } 2.6 \% \text { versus } 4.9 \% ; p=0.04 \\
\text { - } \quad \text { TLF: } 7.5 \% \text { versus } 11.9 \% ; p=0.02\end{array}$ & $\begin{array}{l}\text { Non-inferior } \\
\text { Definite very late } \\
\text { ST, MI, TLR, and } \\
\text { TLF lower with } \\
\text { Orsiro BP-DES } \\
\text { than Xience EES }\end{array}$ \\
\hline BIOSTEM| ${ }^{84}$ & 2019 & $\begin{array}{l}\text { Superiority } \\
\text { RCT }\end{array}$ & 12 & Xience EES & 649 & 651 & $\begin{array}{ll}\text { - } & \text { Definite or probable ST: RR, 0.69; 95\% Crl, } \\
& 0.32-1.69 \\
\text { - } & \text { Ml: RR, } 1.21 ; 95 \% \mathrm{Crl}, 0.60-2.46 \\
\text { - } & \text { Cardiac death: RR, } 0.77 ; 95 \% \mathrm{Crl}, 0.43-1.40 \\
\text { - } & \text { All-cause death: } \mathrm{RR}, 0.97 ; 95 \% \mathrm{Crl}, 0.58-1.62 \\
\text { - } & \text { TLR: RR, } 0.57 ; 95 \% \mathrm{Crl}, 0.28-1.12 \\
\text { - } & \text { TLF: RR, } 0.59 ; 95 \% \mathrm{Crl}, 0.37-0.94\end{array}$ & $\begin{array}{l}\text { Non-superior } \\
\text { TLF lower with } \\
\text { Orsiro BP-DES } \\
\text { than Xience EES }\end{array}$ \\
\hline
\end{tabular}

Values are mean \pm standard deviation or median (interquartile range).

$B P$-DES = biodegradable polymer drug-eluting stents; $C r l=$ credibility interval; $E E S=$ everolimus-eluting stents; $L L L=l$ ate lumen loss; $M I=$ myocardial infarction; $N / A=$ not available; $R C T$ = randomised controlled trial; $R R=$ risk ratio; $S T$ = stent thrombosis; $T L F=$ target lesion failure; $T L R=$ target lesion revascularisation;

$Z E S=$ zotarolimus-eluting stents .

Table 3: Findings of landmark trials related to other types of biodegradable polymer drug-eluting stents

\begin{tabular}{|c|c|c|c|c|c|c|c|c|}
\hline \multirow[t]{2}{*}{ Trial name } & \multirow[t]{2}{*}{ Year } & \multirow{2}{*}{$\begin{array}{l}\text { Follow-up } \\
\text { (months) }\end{array}$} & \multicolumn{2}{|l|}{ Stent type } & \multicolumn{2}{|c|}{ Patients (n) } & \multirow[t]{2}{*}{ Outcomes } & \multirow[t]{2}{*}{ Conclusion } \\
\hline & & & BP-DES & DP-DES & BP-DES & DP-DES & & \\
\hline $\begin{array}{l}\text { Separham } \\
\text { et al. }{ }^{85}\end{array}$ & 2011 & 12 & $\begin{array}{l}\text { BioMatrix } \\
\text { BES }\end{array}$ & $\begin{array}{l}\text { Xience } \\
\text { EES }\end{array}$ & 100 & 100 & $\begin{array}{ll}\text { - } & \text { ST: } 0 \% \text { versus } 0 \% \\
\text { - } & \text { Ml: } 0 \% \text { versus } 0 \% \\
\text { - } & \text { Cardiac death: } 0 \% \text { versus } 0 \%\end{array}$ & Non-inferior \\
\hline Xu et al. ${ }^{86}$ & 2011 & 24 & Tivoli SES & $\begin{array}{l}\text { Endeavor } \\
\text { ZES }\end{array}$ & 168 & 156 & $\begin{array}{l}\text { - } \quad \text { Definite or probable ST: } 0.6 \% \text { versus } 0 \% ; p=1.00 \\
\text { - } \quad \text { Ml: } 2.4 \% \text { versus } 1.3 \% ; p=0.69 \\
\text { - } \quad \text { Cardiac death: } 0.6 \% \text { versus } 0 \% ; p=1.00 \\
\text { - } \quad \text { All-cause death: } 0.6 \% \text { versus } 0 \% ; p=1.00 \\
\text { - } \quad \text { TLR: } 4.2 \% \text { versus } 9.6 \% ; p=0.0495\end{array}$ & $\begin{array}{l}\text { Non-inferior } \\
\text { TLR lower with Tivoli } \\
\text { SES than Endeavour } \\
\text { ZES }\end{array}$ \\
\hline EVOLVE FHU ${ }^{87}$ & 2013 & 24 & $\begin{array}{l}\text { Synergy } \\
\text { EES }\end{array}$ & $\begin{array}{l}\text { Promus } \\
\text { Element } \\
\text { EES }\end{array}$ & 193 & 98 & $\begin{array}{l}\text { - } \quad \text { Definite or probable ST: } 0 \% \text { versus } 0 \% \\
\text { - } \\
\text { - } \text { Ml: } 3.1 \% \text { versus } 0 \% \\
\text { - } \\
\text { - }\end{array}$ & Non-inferior \\
\hline TARGET | 88 & 2013 & 12 & $\begin{array}{l}\text { Firehawk } \\
\text { SES }\end{array}$ & $\begin{array}{l}\text { Xience } \\
\text { EES }\end{array}$ & 227 & 231 & $\begin{array}{l}\text { - } \quad \text { Definite or probable ST: } 0 \% \text { versus } 0 \% \\
\text { - } \quad \text { Ml: } 1.3 \% \text { versus } 2.2 \% ; \mathrm{p}=0.72 \\
\text { - } \quad \text { Cardiac death: } 0.4 \% \text { versus } 0 \% \\
\text { - } \quad \text { All-cause death: } 0.4 \% \text { versus } 0.9 \% ; p=1.00 \\
\text { - } \quad \text { TLR: } 0.4 \% \text { versus } 0.4 \% ; p=1.00\end{array}$ & Non-inferior \\
\hline CENTURY \|\|$^{89}$ & 2014 & 9 & $\begin{array}{l}\text { Ultimaster } \\
\text { SES }\end{array}$ & $\begin{array}{l}\text { Xience } \\
\text { EES }\end{array}$ & 551 & 550 & $\begin{array}{l}\text { - } \quad \text { ST: } 0.9 \% \text { versus } 0.9 \% ; p=0.99 \\
\text { - } \quad \text { Ml: } 2.0 \% \text { versus } 2.7 \% ; p=0.43 \\
\text { - } \quad \text { Cardiac death: } 0.9 \% \text { versus } 1.1 \% ; p=0.76 \\
\text { - } \quad \text { All-cause death: } 1.3 \% \text { versus } 1.6 \% ; p=0.61 \\
\text { - } \quad \text { TLR: } 2.7 \% \text { versus } 2.2 \% ; p=0.56\end{array}$ & Non-inferior \\
\hline LONG-DES V90 & 2014 & 12 & Nobori BES & $\begin{array}{l}\text { Promus } \\
\text { Element } \\
\text { EES }\end{array}$ & 245 & 255 & $\begin{array}{l}\text { - } \quad \text { Definite or probable ST: } 1.2 \% \text { versus } 0 \% ; p=0.12 \\
\text { - } \quad \text { MI: } 13.9 \% \text { versus } 15.7 \% ; p=0.53 \\
\text { - } \quad \text { Cardiac death: } 0.8 \% \text { versus } 0.4 \% ; p=0.62 \\
\text { - } \quad \text { All-cause death: } 0.8 \% \text { versus } 0.4 \% ; p=0.62 \\
\text { - } \quad \text { TLR: } 3.3 \% \text { versus } 2.0 \% ; p=0.44\end{array}$ & Non-inferior \\
\hline $\begin{array}{l}\text { BASKET- } \\
\text { PROVE } \|^{91}\end{array}$ & 2015 & 24 & Nobori BES & $\begin{array}{l}\text { Xience } \\
\text { EES }\end{array}$ & 765 & 765 & $\begin{array}{l}\text { - } \quad \text { Definite or probable ST: } 0.4 \% \text { versus } 0.7 \% ; \\
\quad \mathrm{p}=0.48 \\
\text { - } \quad \text { Ml: } 2.4 \% \text { versus } 2.7 \% ; p=0.64 \\
\text { - } \quad \text { Cardiac death: } 1.3 \% \text { versus } 0.9 \% ; p=0.46 \\
\text { - } \quad \text { All-cause death: } 2.6 \% \text { versus } 2.2 \% ; p=0.61\end{array}$ & Non-inferior \\
\hline
\end{tabular}


Table 3: Cont.

\begin{tabular}{|c|c|c|c|c|c|c|c|c|}
\hline \multirow[t]{2}{*}{ Trial name } & \multirow[t]{2}{*}{ Year } & \multirow{2}{*}{$\begin{array}{l}\text { Follow-up } \\
\text { (months) }\end{array}$} & \multicolumn{2}{|l|}{ Stent type } & \multicolumn{2}{|c|}{ Patients (n) } & \multirow[t]{2}{*}{ Outcomes } & \multirow[t]{2}{*}{ Conclusion } \\
\hline & & & BP-DES & DP-DES & BP-DES & DP-DES & & \\
\hline DESSOLVE $\|^{92}$ & 2015 & 9 & $\begin{array}{l}\text { MiStent } \\
\text { SES }\end{array}$ & $\begin{array}{l}\text { Endeavor } \\
\text { ZES }\end{array}$ & 123 & 61 & $\begin{array}{l}\text { - } \quad \text { ST: } 0.9 \% \text { versus } 1.7 \% \\
\text { - } \quad \text { Ml: } 2.6 \% \text { versus } 3.3 \% \\
\text { - } \quad \text { Cardiac death: } 0.9 \% \text { versus } 1.7 \% \\
\text { - } \quad \text { All-cause death: } 0.9 \% \text { versus } 1.7 \% \\
\text { - } \quad \text { TLR: } 0.9 \% \text { versus } 1.7 \%\end{array}$ & Non-inferior \\
\hline EVERBIO $\|^{93}$ & 2015 & 9 & $\begin{array}{l}\text { BioMatrix } \\
\text { BES }\end{array}$ & $\begin{array}{l}\text { Promus } \\
\text { Element } \\
\text { EES }\end{array}$ & 80 & 80 & 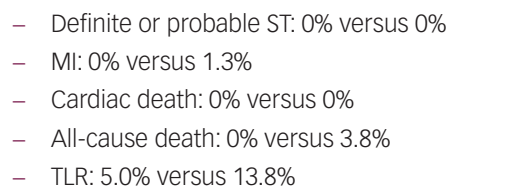 & Non-inferior \\
\hline EVOLVE $\|^{194}$ & 2015 & 12 & $\begin{array}{l}\text { Synergy } \\
\text { EES }\end{array}$ & $\begin{array}{l}\text { Promus } \\
\text { Element } \\
\text { EES }\end{array}$ & 846 & 838 & $\begin{array}{l}\text { - } \quad \text { Definite or probable ST: } 0.4 \% \text { versus } 0.6 \% ; \\
\quad p=0.50 \\
\text { - } \quad \text { MI: } 5.4 \% \text { versus } 5.0 \% ; p=0.68 \\
\text { - } \quad \text { Cardiac death: } 0.5 \% \text { versus } 0.9 \% ; p=0.34 \\
\text { - } \quad \text { All-cause death: } 1.1 \% \text { versus } 1.1 \% ; p=0.95 \\
\text { - } \quad \text { TLR: } 2.6 \% \text { versus } 1.7 \% ; p=0.21\end{array}$ & Non-inferior \\
\hline SORT OUT VI' ${ }^{97}$ & 2015 & 36 & $\begin{array}{l}\text { BioMatrix } \\
\text { BES }\end{array}$ & $\begin{array}{l}\text { Resolute } \\
\text { Integrity } \\
\text { ZES }\end{array}$ & 1,497 & 1,502 & $\begin{array}{l}\text { - } \quad \text { Definite or probable ST: } 1.2 \% \text { versus } 1.3 \% ; \\
\quad \text { - } \quad \text { MI: } 4.7 \% \text { versus } 4.1 \% ; p=0.49 \\
\text { - } \quad \text { Cardiac death: } 3.4 \% \text { versus } 2.7 \% ; p=0.31 \\
\text { - } \quad \text { All-cause death: } 7.6 \% \text { versus } 7.6 \% ; p=0.96 \\
\text { - } \quad \text { TLR: } 5.5 \% \text { versus } 5.4 \% ; p=0.90\end{array}$ & Non-inferior \\
\hline ISAR-TEST $4^{95}$ & 2016 & 60 & $\begin{array}{l}\text { Yukon } \\
\text { Choice PC } \\
\text { SES }\end{array}$ & $\begin{array}{l}\text { Xience } \\
\text { EES }\end{array}$ & 1,299 & 652 & $\begin{array}{l}\text { - } \quad \text { Definite or probable ST: } 1.2 \% \text { versus } 1.4 \% ; \\
\quad p=0.67 \\
\text { - } \quad \text { Ml: } 5.5 \% \text { versus } 5.0 \% ; p=0.67 \\
\text { - } \quad \text { Cardiac death: } 5.2 \% \text { versus } 5.2 \% ; p=0.89 \\
\text { - } \quad \text { All-cause death: } 14.7 \% \text { versus } 14.8 \% ; p=0.95 \\
\text { - } \quad \text { TLR: } 13.9 \% \text { versus } 12.6 \% ; p=0.46\end{array}$ & Non-inferior \\
\hline COMPARE $\|^{96}$ & 2017 & 60 & Nobori BES & $\begin{array}{l}\text { Xience } \\
\text { EES }\end{array}$ & 1,795 & 912 & $\begin{array}{l}\text { - } \quad \text { Definite or probable ST: } 1.7 \% \text { versus 1.6\%; } \\
\quad \text { p=0.96 } \\
\text { - } \quad \text { Ml: } 7.6 \% \text { versus } 7.0 \% ; p=0.56 \\
\text { - } \quad \text { Cardiac death: } 4.6 \% \text { versus 3.9\%; } p=0.45 \\
\text { - } \quad \text { All-cause death: } 8.6 \% \text { versus } 8.2 \% ; p=0.72 \\
\text { - } \quad \text { TLR: } 7.9 \% \text { versus } 7.1 \% ; p=0.47\end{array}$ & Non-inferior \\
\hline DESSOLVE $\|^{198}$ & 2018 & 12 & $\begin{array}{l}\text { MiStent } \\
\text { SES }\end{array}$ & $\begin{array}{l}\text { Xience } \\
\text { EES }\end{array}$ & 703 & 695 & $\begin{array}{l}\text { - } \quad \text { Definite or probable ST: } 0.7 \% \text { versus } 0.9 \% \\
\quad \mathrm{p}=0.76 \\
\text { - } \quad \text { Ml: } 2.4 \% \text { versus } 2.2 \% ; p=0.73 \\
\text { - } \quad \text { Cardiac death: } 2.0 \% \text { versus } 1.6 \% ; p=0.55 \\
\text { - } \quad \text { All-cause death: } 3.6 \% \text { versus } 2.6 \% ; p=0.29 \\
\text { - } \quad \text { TLR: } 3.4 \% \text { versus } 4.1 \% ; p=0.48\end{array}$ & Non-inferior \\
\hline NEXT ${ }^{99}$ & 2018 & 60 & Nobori BES & $\begin{array}{l}\text { Xience } \\
\text { EES }\end{array}$ & 1,283 & 1,285 & $\begin{array}{l}\text { - } \quad \text { Definite or probable ST: } 0.5 \% \text { versus } 0.3 \% ; \\
\quad p=0.52 \\
\text { - } \quad \text { Ml: } 5.2 \% \text { versus } 4.8 \% ; p=0.72 \\
\text { - } \quad \text { Cardiac death: } 4.4 \% \text { versus } 3.9 \% ; p=0.54 \\
\text { - } \quad \text { All-cause death: } 11.7 \% \text { versus } 12.6 \% ; p=0.51 \\
\text { - } \quad \text { TLR: } 9.8 \% \text { versus } 9.3 \% ; p=0.79\end{array}$ & Non-inferior \\
\hline $\begin{array}{l}\text { TARGET All } \\
\text { Comers }\end{array}$ & 2018 & 12 & $\begin{array}{l}\text { Firehawk } \\
\text { SES }\end{array}$ & $\begin{array}{l}\text { Xience } \\
\text { EES }\end{array}$ & 823 & 830 & $\begin{array}{l}\text { - } \quad \text { Definite or probable ST: } 1.3 \% \text { versus } 1.3 \% ; \\
\quad p=0.99 \\
\text { - } \quad \text { Ml: } 5.4 \% \text { versus } 4.8 \% ; p=0.62 \\
\text { - } \quad \text { Cardiac death: } 1.2 \% \text { versus } 0.9 \% ; p=0.60 \\
\text { - } \quad \text { All-cause death: } 2.2 \% \text { versus } 2.2 \% ; p=0.98 \\
\text { - } \quad \text { TLR: } 2.0 \% \text { versus } 3.0 \% ; p=0.20\end{array}$ & Non-inferior \\
\hline TALENT ${ }^{101}$ & 2019 & 12 & $\begin{array}{l}\text { Supraflex } \\
\text { SES }\end{array}$ & $\begin{array}{l}\text { Xience } \\
\text { EES }\end{array}$ & 720 & 715 & $\begin{array}{l}\text { - } \quad \text { Definite or probable ST: } 0.8 \% \text { versus } 0.9 \% ; \\
\quad \text { - } \quad \text { MI: } 3.1 \% \text { versus } 3.7 \% ; p=0.55 \\
\text { - } \quad \text { Cardiac death: } 1.0 \% \text { versus } 0.3 \% ; p=0.10 \\
\text { - } \quad \text { All-cause death: } 2.0 \% \text { versus } 0.6 \% ; p=0.02 \\
\text { - } \quad \text { TLR: } 3.5 \% \text { versus } 4.3 \% ; p=0.50\end{array}$ & $\begin{array}{l}\text { Non-inferior } \\
\text { All-cause death } \\
\text { higher with Supraflex } \\
\text { SES than Xience EES }\end{array}$ \\
\hline
\end{tabular}


regions of increased stress during stent expansion, while providing higher drug capacity on the abluminal side than the luminal side.68 The inner layer contains a proBIO passive coating (Biotronik, Bülach, Switzerland) that eliminates the interaction between the metal alloy and surrounding tissue by covering the entire stent surface. ${ }^{69}$ The proBIO passive coating is made from a thin layer $\left(80 \mathrm{~nm}\right.$ ) of silicon carbide. ${ }^{70}$ The silicon carbide has semi-conductor properties that provide a diffusion barrier between the metal ions and cellular proteins to reduce thrombogenicity and promote re-endothelialisation. ${ }^{71}$

\section{Pre-clinical studies}

The unique features of Orsiro BP-DES have been investigated in animal studies. An in vivo study involving a porcine model of coronary stent implantation showed that biodegradable polymer SES had significantly reduced neointimal area $\left(1.8 \pm 2.2\right.$ versus $\left.3.0 \pm 1.5 \mathrm{~mm}^{2} ; \mathrm{p}=0.01\right)$ and area stenosis $(26.4 \pm 15.2 \%$ versus $40.1 \pm 19.5 \% ; \mathrm{p}=0.04)$ at 180 days compared with durable polymer SES..$^{72}$ An ex vivo study involving a porcine carotid to jugular arteriovenous shunt model found that Orsiro BP-DES and Xience EES (the latter manufacturerd by Abbott Vascular, Santa Clara, CA, USA) conferred equivocal thrombogenicity profiles, with comparable fluorescence positive area (mean difference, 2.95; 95\% confidence interval $[\mathrm{Cl}],-1.26$ to $7.15 ; \mathrm{p}=0.286) .{ }^{73}$ The fluorescence positive area examined under confocal microscopy corresponds to the extent of platelet aggregation. ${ }^{74}$

\section{Clinical studies}

The safety and efficacy profiles of Orsiro BP-DES have been evaluated in various studies. Table 2 summarises the findings of clinical studies related to Orsiro BP-DES. ${ }^{56,75-84}$ Overall, the randomised trials showed that Orsiro BP-DES were non-inferior to second-generation DP-DES, including Xience EES and Resolute Integrity ${ }^{\top M}$ ZES (the latter manufacturerd by Medtronic, Santa Rosa, CA, USA). The FDA approved the use of Orsiro BP-DES in patients with CAD based on promising results in recent trials, such as
BIOFLOW V ${ }^{83}$ and BIOSTEMI. ${ }^{84}$ Both of these trials found that Orsiro BP-DES had a significantly reduced risk of target lesion failure, which is a composite of $\mathrm{Ml}$, cardiac death, and TLR, compared with Xience EES. Table 3 summarises the findings of landmark trials related to other types of BP-DES. ${ }^{85-101}$ Overall, the randomised trials showed that other types of BP-DES were also non-inferior to second-generation DP-DES. These devices included Nobori BP-DES and Synergy ${ }^{\mathrm{TM}}$ BP-DES (the latter manufacturerd by Boston Scientific, Marlborough, MA, USA).

A recent meta-analysis of 11,176 patients found that Orsiro BP-DES and second-generation DP-DES conferred equivocal safety and efficacy profiles, with comparable rates of definite or probable ST (odds ratio [OR], 0.77; 95\% Cl, 0.53-1.12; $\mathrm{p}=0.18)$, MI (OR, 0.79; 95\% Cl, 0.63-1.00; $\mathrm{p}=0.05)$, all-cause death $(\mathrm{OR}, 1.17 ; 95 \% \mathrm{Cl}$, $0.84-1.64)$, and target lesion failure (OR, $0.87 ; 95 \% \mathrm{Cl}, 0.72-1.05 ; \mathrm{p}=0.16) .{ }^{102}$ These findings offered reassurance about the clinical performance of Orsiro BP-DES. However, this meta-analysis did not include the recent BIOSTEMI trial, which would have provided the latest clinical evidence to date. ${ }^{84}$

\section{Conclusion}

Ultrathin Orsiro BP-DES showcase the latest innovations in coronary stent technology. The thin-strut cobalt-chromium stent platform with a biodegradable polymer coating enhances biocompatibility by eliminating the potential stimulus for a chronic inflammatory response in the vessel wall. The latest clinical evidence at the time of writing demonstrated that Orsiro BP-DES conferred comparable safety and efficacy profiles to the current gold standard second-generation DP-DES. In light of the recent FDA approval, Orsiro BP-DES represents a suitable alternative to the second-generation DP-DES in patients with CAD. Further randomised trials with greater length of follow-up and larger patient populations are warranted to establish the purported benefits of Orsiro BP-DES. $\square$
1. Nowbar AN, Gitto M, Howard JP, et al. Mortality from ischemic heart disease. Circ Cardiovasc Qual Outcomes. 2019;12:e005375.

2. Kobo $\mathrm{O}$, Roguin A. Orsiro: ultrathin bioabsorbable polymer sirolimus-eluting stent. Future Cardiol. 2019:15:295-300.

Chen $\mathrm{D}$, Jepson $\mathrm{N}$. Coronary stent technology: a narrative review. Med J Aust. 2016:205:277-81.

4. Mueller RL, Sanborn TA. The history of interventional cardiology: cardiac catheterization, angioplasty, and related interventions. Am Heart J. 1995;129:146-72.

5. Castaneda WR, Formanek A, Tadavarthy M, et al. The mechanism of balloon angioplasty. Radiology. 1990;135:565-7

6. McBride W, Lange RA, Hillis LD. Restenosis after successfu coronary angioplasty. Pathophysiology and prevention. N Engl J Med. 1988;318:1734-7.

. Dash A. Complications of coronary intervention: abrupt closure dissection, perforation. Heart Asia. 2013;5:61-5.

8. Lincoff AM, Popma JJ, Ellis SG, et al. Abrupt vessel closure complicating coronary angioplasty: clinical, angiographic and therapeutic profile. J Am Coll Cardiol. 1992:19:926-35.

9. Birkenhauer $P$ Yang $Z$ Gander $B$. Preventing restenosis in early drug-eluting stent era: recent developments and future early drug-eluting stent era: recent developments and

10. Hirshfeld JW, Schwartz JS, Jugo R, et al. Restenosis after coronary angioplasty: a multivariate statistical model to relate lesion and procedure variables to restenosis. The M-HEART Investigators. J Am Coll Cardiol. 1991; 18:647-56.

11. Rousseau H, Puel J, Joffre F, et al. Self-expanding endovascular prosthesis: an experimental study. Radiology. 1987;164:709-14.

12. Sigwart U, Puel J, Mirkovitch V, et al. Intravascular stents to prevent occlusion and restenosis after transluminal angioplasty. N Engl J Med. 1987;316:701-6.

13. de Feyter PJ, de Jaegere PP, Serruys PW. Incidence, predictors, and management of acute coronary occlusion after coronary angioplasty. Am Heart J. 1994;127:643-51.

14. Marrocco CJ, Jaber R, White RA, et al. Intravascular ultrasound. Semin Vasc Surg. 2012;25:144-52.

15. Maehara A, Ben-Yehuda O, Ali Z, et al. Comparison of stent expansion guided by optical coherence tomography versus intravascular ultrasound: the ILUMIEN II study (observational study of optical coherence tomography [OCT] in patients study of optical coherence tomography [OCT] in patients undergoing fractional flow reserve [FFR] and percutaneous
coronary intervention). JACC Cardiovasc Interv. 2015;8:1704-14

16. Htay T, Liu MW. Drug-eluting stent: a review and update. Vasc Health Risk Manag. 2005;1:263-76.
17. Serruys PW, de Jaegere PP, Kiemeneij F, et al. A comparison of balloon-expandable-stent implantation with balloon angioplasty in patients with coronary artery disease. Benestent Study Group. N Engl J Med. 1994;331:489-95.

18. Curcio A, Torelia D, Idolfi C. Mechanisms of smooth muscle cel proliferation and endothelial regeneration after vascular injury proliferation and endothelial regeneration after vascular injury

19. Sousa JE, Serruys PW, Costa MA. New frontiers in cardiology: drug-eluting stents: Part I. Circulation. 2003:107:2274-9.

20. Buccheri D, Piraino D, Andolina G, Cortese B. Understanding and managing in-stent restenosis: a review of clinical daya, from pathogenesis to treatment. J Thorac Dis. 2016;8:e1150-e62.

21. Martin DM, Boyle FJ. Drug-eluting stents for coronary artery disease: a review. Med Eng Phys. 2011;33:148-63

22. Sousa JE, Serruys PW, Costa MA. New frontiers in cardiology: drug-eluting stents: Part II. Circulation 2003;107:2383-9.

23. Abizaid A. Sirolimus-eluting coronary stents: a review. Vasc Health Risk Manag. 2007;3:191-201.

24. Woods TC, Marks AR. Drug-eluting stents. Annu Rev Med. 2004:55:169-78.

25. Lemos PA, Serruys PW, Sousa JE. Drug-eluting stents: cost versus clinical benefit. Circulation. 2003:107:3003-7.

26. Versaci $F$ Gaspardone A Tomai $F$ et al A comparison of coronary artery stenting with angioplasty for isolated stenosis of the proximal left anterior descending artery: five year clinica follow up. Heart. 2004;90:672-5.

27. Morice MC, Serruys PW, Sousa JE, et al. A randomized comparison of a sirolimus-eluting stent with a standard stent for coronary revascularization. N Eng/ J Med. 2002;346:1773-80.

28. Grube E, Silber S, Hauptmann KE, et al. TAXUS I: six- and twelve-month results from a randomized, double-blind trial on a slow-release paclitaxel-eluting stent for de novo coronary lesions. Circulation. 2003;107:38-42.

29. Mauri L, Orav EJ, Candia SC, et al. Robustness of late lumen loss in discriminating drug-eluting stents across variable observational and randomized trials. Circulation. variable observationa

30. Mauri L Orav EJ, Kuntz RE. Late loss in lumen diameter and binary restenosis for drug-eluting stent comparison. Circulation 2005:111:3435-42.

31. Butany J, Carmichael K, Leong SW, Collins MJ. Coronary artery stents: identification and evaluation. J Clin Pathol. 2005;58:795-804
32. Cutlip DE, Windecker S, Mehran R, et al. Clinical end points in coronary stent trials' a case for standardized definitions. Circulation. 2007;115:2344-51.

33. Byrne RA, Joner M, Kastrati A. Stent thrombosis and restenosis: what have we learned and where are we going? The Andreas Grüntzig Lecture ESC 2014. Eur Heart J. 2015;36:3320-31.

34. Joner M, Finn AV, Farb A, et al. Pathology of drug-eluting stents in humans: delayed healing and late thrombotic risk. J Am Coll Cardiol. 2006:48:193-202.

35. Nakazawa G, Finn AV, Virmani R. Vascular pathology of drug-eluting stents. Herz. 2007;32:274-80.

36. Lüscher TF, Steffel J, Eberli FR, et al. Drug-eluting stent and coronary thrombosis: biological mechanisms and clinical implications. Circulation. 2007;115:1051-8.

37. Stone GW, Moses JW, Ellis SG, et al. Safety and efficacy of sirolimus- and paclitaxel-eluting coronary stents. N Eng/ I Med. 2007;356:998-1008

38. Bavry AA, Kumbhani DJ, Helton TJ, et al. Late thrombosis of drug-eluting stents: a meta-analysis of randomized clinica trials. Am J Med. 2006:119:1056-61.

39. Holmes DR, Kereiakes DJ, Garg $\mathrm{S}$, et al. Stent thrombosis. J Am Coll Cardiol. 2010;56:1357-65.

40. Brugaletta S, Burzotta F Sabaté M. Zotarolimus for the treatment of coronary artery disease: pathophysiology, DES design, clinical evaluation and future perspective. Expert Opin Pharmacother. 2009;10:1047-58.

41. Smits PC, Vlachojannis GJ, MCFadden EP, et al. Final 5-year follow-up of a randomized controlled trial of everolimus- and paclitaxel-eluting stents for coronary revascularization in daily practice: the COMPARE trial (a trial of everolimus-eluting stents and paclitaxel stents for coronary revascularization in daily practice). JACC Cardiovasc Interv. 2015;8:1157-65.

42. Kirtane AJ, Leon MB, Ball MW, et al. The "final" 5-year follow-up from the ENDEAVOR IV trial comparing a zotarolimus-eluting stent with a paclitaxel-eluting stent. JACC CardiovasC Interv. 2013;6:325-33.

43. Qian F, Zhong Y, Hannan EL. Long-term comparative effectiveness of Endeavor Zotarolimus-eluting and everolimus-eluting stents in New York. Int I Cardiol. 2017:241:437-43.

44. Neumann FJ, Sousa-Uva M, Ahlsson A, et al. 2018 ESC/EACTS guidelines on myocardial revascularization. Eur Heart $\lrcorner$. 2019;40:87-165

45. Zocca P, Kok MM, Tandjung K, et al. 5-year outcome following randomized treatment of all-comers with zotarolimus-eluting 
Resolute Integrity and everolimus-eluting PROMUS Element coronary stents: final report of the DUTCH PEERS (TWENTE II) trial. JACC Cardiovasc Interv. 2018;11:462-9.

46. Tong R, Gabrielson NP, Fan TM, Cheng J. Polymeric nanomedicines based on poly(lactide) and poly(lactide-co-glycolide). Curr Opin Solid State Mater Sci. 2013;16:323-32.

47. Goyal BK, Kalmath BC, Kawar R, et al. Experience with BioMatrix $\mathrm{BES}$ and other DES in all-comers setting: a retrospective overview. Indian Heart J. 2013;65:678-82.

48. Ostojic M, Sagic D, Jung R, et al. The pharmacokinetics of biolimus A9 after elution from the Nobori stent in patients with coronary artery disease: the NOBORI PK study. Catheter Cardiovasc Interv. 2008;72:901-8.

49. Kolandaivelu K, Swaminathan R, Gibson WJ, et al. Stent thrombogenicity early in high-risk interventional settings is driven by stent design and deployment and protected by polymer-drug coatings. Circulation. 2011:123:1400-9.

50. Soucy NV, Feygin JM, Tunstall R, et al. Strut tissue coverage and endothelial cell coverage: a comparison between bare metal stent platforms and platinum chromium stents with and without everolimus-eluting coating. Eurolntervention. 2010;6:630-7.

51. Farb A, Weber DK, Kolodgie FD, et al. Morphological predictors of restenosis after coronary stenting in humans. Circulation. 2002;105:2974-80

52. Kastrati A, Mehilli J, Dirschinger J, et al. Intracoronary stenting and angiographic results: strut thickness effect on restenosis outcome (ISAR-STEREO) trial. Circulation. 2001;103:2816-21.

53. Nikam N, Steinberg TB, Steinberg DH. Advances in stent technologies and their effect on clinical efficacy and safety. Med Devices (AucKI). 2014;7:165-78

54. Khan W, Farah S, Domb AJ. Drug eluting stents: developments and current status. J Control Release. 2012;161:703-12

55. Schurtz G, Delhaye C, Hurt C, et al. Biodegradable polymer Biolimus-eluting stent (Nobori) for the treatment of coronary artery lesions: review of concept and clinical results. Med Devices (Auckl). 2014;7:35-43

56. Jensen LO, Thayssen P, Maeng M, et al. Randomized comparison of a biodegradable polymer ultrathin strut sirolimus-eluting stent with a biodegradable polymer biolimus-eluting stent in patients treated with percutaneous coronary intervention: the SORT OUT VII trial. Circ Cardiovasc Interv. 2016;9:e003610

57. Barragan P, Sainsous J, Silvestri M, et al. Ticlopidine and subcutaneous heparin as an alternative regimen following coronary stenting. Cathet Cardiovasc Diagn. 1994;32:133-8.

Levine GN, Bates ER, Bittl JA, et al. 2016 ACC/AHA guideline focused update on duration of dual antiplatelet therapy in patients with coronary artery disease: a report of the American College of Cardiology/American Heart Association Task Force on clinical practice guidelines Circulation. 2016;134:e123-55

59. Nakamura $\mathrm{M}$, lijima R, Ako J, et al. Dual antiplatelet threapy for 6 versus 18 months after biodegradable polymer drug-eluting stent implantation. JACC Cardiovasc Interv. 2017;10:1189-98.

60. Tittelbach M, Diener T. Orsiro - the first hybrid drug-eluting stent, opening up a new class of drug-eluting stents for superior patient outcomes. ICR. 2011;6:142-4.

61. Ismail MD, Ahmad WAW, Leschke M, et al. The outcomes of patients with very small coronary artery disease treated with thin strut cobalt chromium bare metal stents: an observationa study. Springerplus. 2016;5:1668

62. Jorge C, Dubois C. Clinical utility of platinum chromium bare-metal stents in coronary heart disease. Med Devices (Auckl). 2015:8:359-67.

63. Iglesias JF, Roffi M, Degrauwe S, et al. Orsiro cobalt-chromium sirolimus-eluting stent: present and future perspectives. Expert Rev Med Devices. 2017;14:773-88.

64. Lee $\mathrm{D}$, de la Torre Hernandez JM. The newest generation of drug-eluting stents and beyond. Eur Cardiol. 2018;13:54-9.

65. Simamora P, Chern W. Poly-L-lactic acid: an overview. J Drugs Dermatol. 2006;5:436-40.

66. Lee JM, Park S, Lim SY, et al. Angiographic and clinical comparison of novel Orsiro Hybrid sirolimus-eluting stents and Resolute Integrity zotarolimus-eluting stents in all-comers with coronary artery disease (ORIENT trial): study protocol for randomized controlled trial. Trials. 2013;14:398.

67. Bangalore S, Toklu B, Patel N, et al. Newer-generation ultrathin strut drug-eluting stents versus older second-generation thicker strut drug-eluting stents for coronary artery disease. Circulation. 2018:138:2216-26.

68. Cassese S, Lahmann AL, Joner M. Ultrathin strut biodegradable-polymer sirolimus-eluting stents: being wary or going with the flow? J Thorac Dis. 2018;10:688-92.

69. Amon M, Bolz A, Schaldach M. Improvement of stenting therapy with a silicon carbide coated tantalum stent. J Mater Sci Mater Med. 1996:7:273-8.

70. Dahm JB, Willems T, Wolpers $\mathrm{HG}$, et al. Clinical investigation into the observation that silicon carbide coating on cobalt chromium stents leads to early differentiating functional endothelial layer, increased safety and DES-like recurren stenosis rates: results of the PRO-Heal Registry (PRO-Kinetic enhancing rapid in-stent endothelialisation). Eurolntervention 2009; 4:502-8.

71. Kalnins U, Erglis A, Dinne I, et al. Clinical outcomes of silicon carbide coated stents in patients with coronary artery disease. Med Sci Monit. 2002;8:PI16-P20.

72. Koppara T, Joner M, Bayer G, et al. Histopathological comparison of biodegradable polymer and permanent polymer based sirolimus eluting stents in a porcine model of coronary stent implantation. Thromb Haemost. 2012;107:1161-71.

73. Otsuka F, Cheng Q, Yahagi $K$, et al. Acute thrombogenicity of a durable polymer everolimus-eluting stent relative to contemporary drug-eluting stents with biodegradable polyme coatings assessed ex vivo in a swine shunt model. JACC Cardiovasc Intery 2015:8:1248-60.

74. Peerschke El. Bound fibrinogen distribution on stimulated platelets. Examination by confocal scanning laser microscopy. Am J Pathol. 1995;147:678-87.

75. Hamon M, Niculescu R, Deleanu D, et al. Clinical and angiographic experience with a third-generation drug-eluting Orsiro stent in the treatment of single de novo coronary artery lesions (BIOFLOW-1): a prospective, first-in-man study. Eurolntervention. 2013;8:1006-11.

76. Windecker S, Haude M, Neumann FJ, et al. Comparison of a novel biodegradable polymer sirolimus-eluting stent with a durable polymer everolimus-eluting stent: results of the randomized BIOFLOW-II trial. Circ Cardiovasc Interv. of the randomized

77. Waltenberger J, Brachmann J, van der Heyden J, et al. Real-world experience with a novel biodegradable polyme sirolimus-eluting stent: twelve-month results of the BIOFLOW-II registry. Eurolntervention. 2016;11:1106-10.

78. Kang SH, Chung WY, Lee JM, et al. Angiographic outcomes of Orsiro biodegradable polymer sirolimus-eluting stents and Resolute Integrity durable polyme zotarolimus-eluting stents: results of the ORIENT trial. Eurolntervention. 2017;12:1623-31.

79. Teeuwen K, van der Schaaf RJ, Adriaenssens T, et al. Randomized multicenter trial investigating angiographic outcomes of hybrid sirolimus-eluting stents with biodegradable polymer compared with everolimus-eluting stents with durable polymer in chronic total occlusions: the PRISON IV trial. JACC polymer in chronic total occlusions:
Cardiovasc Interv. 2017:10:133-43.

80. Pilgrim T, Piccolo R Heg D, et al Ultrathin-strut, biodegradable-polymer, sirolimus-eluting stents versus thin-strut, durable-polymer, everolimus-eluting stents for percutaneous coronary revascularisation: 5-year outcomes of the BIOSCIENCE randomised trial. Lancet. 2018;392:737-46.

81. Kok MM, Zocca P, Buiten RA, et al. Two-year clinical outcome of all-comers treated with three highly dissimilar contemporary coronary drug-eluting stents in the randomised BIO-RESORT trial. Eurolntervention. 2018;14:915-23.

82. von Birgelen C, Zocca P, Buiten RA, et al. Thin composite wire strut, durable polymer-cuated (Resolute Onyx) versus ultrathin cobalt-chromium, bioresorbable polymer-coated (Orsiro) drug-eluting stents in allcomers with coronary artery disease (BIONYX): an international, single-blind, randomised non-inferiority trial. Lancet. 2018;392:1235-45.

83. Kandzari DE Koolen IJ, Doros $\mathrm{G}$, et al. Ultrathin bioresorbable polymer sirolimus-eluting stents versus thin durable polymer everolimus-eluting stents. J Am Coll Cardiol. 2018:72:3287-97.

84. Iglesias J, Muller O, Heg D, et al. Biodegradable polymer sirolimus-eluting stents versus durable polymer everolimus-eluting stents in patients with ST-segment elevation myocardial infarction (BIOSTEMI): a single-blind, prospective, randomised superiority trial. Lancet. 2019;394:1243-53.

85. Separham A, Sohrabi B, Aslanabadi N, Ghaffari S. The twelve-month outcome of biolimus-eluting stent with biodegradable polymer compared with an everolimus-eluting stent with durable polymer. I Cardiovasc Thorac Res. 2011;3:113-6

86. XU B, Dou K, Han Y et al. A prospective multicenter parallel-controlled trial of TIVOLI biodegradable-polymer-based sirolimus-eluting stent compared to ENDEAVOR zotarolimus-eluting stent for the treatment of coronary artery disease: 8-month angiographic and 2-year clinical follow-up results. Chin Med J. 2011:124:811-6.

87. Meredith IT, Verheye S, Weissman NJ, et al. Six-month IVUS and two-year clinical outcomes in the EVOLVE FHU trial: a randomised evaluation of a novel bioabsorbable polymer-coated, everolimus-eluting stent. Eurolntervention. 2013;9:308-15.

88. Gao RL, XU B, Lansky AJ, et al. A randomised comparison of a novel abluminal groove-filled biodegradable polymer sirolimus-eluting stent with a durable polymer everolimus-eluting stent: clinical and angiographic follow-up of the TARGET I trial. Eurolntervention. 2013;9:75-83.

89. Saito $\mathrm{S}$, Valdes-Chavarri M, Richardt $\mathrm{G}$, et al. A randomized, prospective, intercontinental evaluation of a bioresorbable polymer sirolimus-eluting coronary stent system: the CENTURY II (Clinical Evaluation of New Terumo Drug-Eluting Coronary Stent System in the Treatment of Patients with Coronary Artery Disease) trial. Eur Heart J. 2014;35:2021-31.

90. Lee JY, Park DW, Kim YH, et al. Comparison of biolimus A9-eluting (Nobori) and everolimus-eluting (Promus Element) stents in patients with de novo native long coronary artery lesions: a randomized Long Drug-Eluting Stent V trial. Circ Cardiovasc Interv 2014:7:322-9.

91. Kaiser $\mathrm{C}$, Galatius $\mathrm{S}$, Jeger $\mathrm{R}$, et al. Long-term efficacy and safety of biodegradable-polymer biolimus-eluting stents: main results of the Basel Stent Kosten-Effektivitats Trial-PROspective Validation Examination II (BASKET-PROVE II), a randomized, controlled noninferiority 2-year outcome trial. Circulation. 2015;131:74-81

92. Wijns W, Vrolix M, Verheye S, et al. Randomised study of a bioabsorbable polymer-coated sirolimus-eluting stent: results of the DESSOLVE II trial. Eurolntervention. 2015;10:1383-90.

93. Puricel S, Arroyo D, Corpataux N, et al. Comparison of everolimus- and biolimus-eluting coronary stents with everolimus-eluting bioresorbable vascular scaffolds. J Am Coll Cardiol. 2015;65:791-801.

94. Kereiakes DJ, Meredith IT, Windecker S, et al. Efficacy and safety of a novel bioabsorbable polymer-coated everolimus-eluting coronary stent: the EVOLVE II randomized trial. Circ Cardiovasc Interv. 2015;8:e002372.

95. Kufner S, Byrne RA, Valenskini M, et al. Five-year outcomes from a trial of three limus-eluting stents with different polymer coating in patients with coronary artery disease: final results from the ISAR-TEST 4 randomised trial. Eurolntervention. 2016;11:1372-9.

96. Vlachojannis GJ, Smits PC, Hofma SH, et al. Biodegradable polymer biolimus-eluting stents versus durable polymer everolimus-eluting stents in patients with coronary artery disease: final 5-year report from the COMPARE II trial (abluminal biodegradable polymer biolimus-eluting stent versus durable polymer everolimus-eluting stent). JACC CardiovasC Interv. 2017:10:1215-21.

97. Raungaard B, Christiansen EH, Bøtker HE, et al. Comparison of durable-polymer zotarolimus-eluting and biodegradable-polymer biolimus-eluting coronary stents in patients with coronary artery disease: 3 -year clinical outcomes in the randomized SORT OUT VI trial. JACC Cardiovasc Interv. 2017;10:255-64

98. de Winter RJ, Katagiri Y, Asano T, et al. A sirolimus-eluting bioabsorbable polymer-coated stent (Mistent) versus an everolimus-eluting durable polymer stent (Xience) after percutaneous coronary intervention (DESSOLVE III): a randomised, single-blind, multicentre, non-inferiority, phase 3 trial. Lancet. 2018;391:431-40.

99. Natsuaki M, Kozuma K, Morimoto T, et al. Five-year outcome of a randomized trial comparing second generation drug-eluting stents using either biodegradable polymer or durable polymer: the NOBORI biolimus-eluting versus XIENCE/PROMUS everolimus-eluting stent trial (NEXT). XIENCE/PROMUS everolimus-eluting

100. Lansky A, Wijns W, Xu B, et al. Targeted therapy with a localised abluminal groove, low-dose sirolimus-eluting, biodegradable polymer coronary stent (TARGET All Comers): a multicentre, open-label, randomised non-inferiority trial. Lancet. 2018;392:1117-26

101. Zaman A, de Winter RJ, Kogame N, et al. Safety and efficacy of a sirolimus-eluting coronary stent with ultra-thin strut for treatment of atherosclerotic lesions (TALENT): a prospective multicentre randomised controlled trial. Lancet. 2019;393:987-97.

102. Lipinski MJ, Forrestal BJ, Iantorno M, et al. A comparison of the ultrathin Orsiro Hybrid sirolimus-eluting stent with contemporary drug-eluting stents: a meta-analysis of randomized controlled trials. Cardiovasc Revasc Med. 2018;19:5-11. 